\title{
Desequilibrio y tendencia al equilibrio en la teoría de Walras: Hipótesis implícita de información limitada
}

\author{
José Guillermo Peláez Gramajo*
}

\section{RESUMEN}

El objetivo de este ensayo es examinar, en sus méritos y limitaciones, una de las formas del desequilibrio económico de la teoría de Walras, la no unicidad de precios para cada mercancía expresada en su teoría del arbitraje, que lamentablemente constituye el rasgo del desequilibrio menos conocido. Este ensayo propone que existe en Walras implícitamente una hipótesis de información limitada, la cual le permite explicar el equilibrio imperfecto, previo al examen que él hace del arbitraje y a la formulación de su teoría del numerario.

Palabras clave: no unicidad de precios, arbitraje, numerario, equilibrio, equilibrio imperfecto, desequilibrio.

Clasificación JEL: B13, D50, D51.

\begin{abstract}
The aim of this essay is to analyze, in its merits and limitations, one of the forms of Walras' economic disequilibrium theory, namely, the lack of price uniqueness for each commodity, expressed in his theory of the arbitrage, unfortunately the lesser known-characteristic of disequilibrium. This essay proposes that in Walras exist an implicit hypothesis of limited information, which allows him explaining the imperfect equilibrium, before his examination of the arbitrage and his theory of the numerarie as well.
\end{abstract}

Key words: lack of price uniqueness, arbitrage, numerarie, equilibrium, imperfect equilibrium, disequilibrium.

Classification JEL: B13, D50, D51.

* Profesor-investigador de la Universidad Autónoma Metropolitana, Unidad Xochimilco. 


\section{INTRODUCCIÓN}

En las sociedades capitalistas, en particular en el ámbito de las finanzas y la economía, tradicionalmente se ha invocado la intuición económica, de acuerdo con la cual en una economía de libre empresa existe una coherencia entre la gran magnitud de decisiones de agentes individuales aparentemente independientes, y la compra y venta de mercancías, lo cual, se arguye, desemboca en un equilibrio entre cantidades de bienes y servicios que los consumidores desean comprar y las cantidades de dichos bienes y servicios que los productores quieren vender. El argumento es que, por un lado, los consumidores potenciales normalmente podrán alcanzar sus planes de compra y, por otro, los oferentes potenciales comúnmente no planearán la producción de una vasta cantidad de mercancías que no puedan vender. Esta idea de equilibrio entre oferta y demanda en cada uno de los mercados de la economía es tan generalizada que se da por sentada y no existe, incluso en el ambiente intelectual, mayor inquietud respecto del mecanismo teórico que explique cómo ocurre este equilibrio.

Sin embargo, la manera en que pudiera manifestarse esta coordinación ha sido una preocupación central de la economía política desde la publicación de La riqueza de las naciones de Adam Smith. En este ensayo nos ocuparemos de un autor, Marie-Esprit Leon Walras (1834-1910), quien propuso una idea teórica razonablemente coherente al respecto, al sugerir, siguiendo a Smith y Cournot, que el hecho de que todos los compradores y vendedores confronten el mismo vector de precios, proporciona la información que se requiere para la coordinación de la existencia del equilibrio y la estabilidad del mismo.

En la teoría propuesta por Walras, la ausencia de equilibrio económico se manifiesta de dos formas, a saber: la no unicidad de precios para cada mercancía, por un lado, e incompatibilidad de planes de ofertas y demandas individuales por otro. Lamentablemente, el estudio de una economía con estas características, en particular en lo que concierne al problema de la ausencia de unicidad del precio para cada mercancía, constituye el rasgo del desequilibrio menos conocido, el cual no ha sido atendido por la teoría económica, con excepción de L. Walras y S. Jevons. ${ }^{1}$ El estudio de la segunda característica del desequilibrio hace referencia al problema de la estabilidad, cuyo estudio corresponde al modelo del

\footnotetext{
${ }^{1}$ Para atender el problema de la unicidad del precio para cada mercancía, Walras propuso la ley del numerario, que analizaremos en este ensayo. Por su parte, Jevons propuso en este mismo contexto la ley de indiferencia, la cual estudiaremos en un futuro trabajo.
} 
tanteo, el cual ha sido ampliamente estudiado en la bibliografía neoclásica, por ejemplo: Arrow y Hurwicz (1958), Arrow, Block y Hurwicz (1959), Arrow y Hahn (1971), Hahn (1982), Hicks (1946), Samuelson (1947).

En efecto, en otro ensayo (Peláez, 2004) hemos analizado el modelo del tanteo walrasiano, el más difundido en la bibliografía, el cual constituye la base del modelo que estudia la estabilidad del equilibrio general en la teoría neoclásica. En este trabajo nos ocuparemos del modelo que propone Walras para examinar el problema del desequilibrio, expresado en la teoría del arbitraje (más de un precio para cada mercancía), cuyos resultados son la existencia del equilibrio general y la ley del numerario.

No pretendemos resolver los problemas que Walras dejó abiertos al enunciar su teoría del numerario. Nuestra intención es hacer un análisis crítico de esta teoría con el objeto de tener una mejor comprensión de sus alcances, limitaciones y su vigencia. Al respecto, podemos señalar que Walras efectuó un estudio formal que hoy consideramos débil, tanto para la existencia ${ }^{2}$ como para la estabilidad del equilibrio general de precios. En gran medida, Walras efectúa un análisis cualitativo que elimina el desequilibrio de los mercados (desigualdad entre oferta y demanda de éstos). Para tal efecto, este autor asume que los precios se ajustan en el buen sentido a partir de la competencia, es decir, los precios tienden a subir en aquellos mercados que manifiestan exceso de demanda y tienden a bajar en los mercados que exhiben exceso de oferta. ${ }^{3}$ Con esta idea, Walras intenta demostrar la existencia de un equilibrio general del sistema económico, tal que todos los precios quedan expresados en términos de un bien común llamado numerario. Sin embargo, lo que realmente se propone es un modelo que carece de generalidad, pues dada la debilidad del estudio axiomático respecto de

\footnotetext{
${ }^{2}$ En el caso de la existencia hay que señalar, en descargo de Walras, que él formuló un sistema de mercados con $k$ mercancías, donde son determinados $k$-1 precios relativos y donde hay $k-1$ ecuaciones de equilibrio que establecen, en cada mercado, la igualdad entre oferta y demanda. Puesto que el número de ecuaciones es igual al número de incógnitas, Walras pretendió demostrar la existencia del equilibrio general aduciendo la existencia de una solución donde todas las ecuaciones estaban solucionadas. Muy pronto se descubrió que existía una falla en la proposición de Walras, pues un supuesto decisivo para la demostración de la existencia es que las funciones de exceso de demanda sean continuas. Dicha demostración fue proporcionada en 1954 por K. Arrow y G. Debreu.

${ }^{3}$ John Hicks (1946, cap. V, pp. 62-77) analizó las condiciones para la estabilidad de una economía de dos bienes propuesta por Walras y generalizó esas condiciones para el caso de una economía de múltiples bienes. En términos técnicos, la condición de equilibrio perfectamente estable de Hicks establece la alternancia del signo de los menores principales de la matriz jacobiana proveniente de las funciones de exceso de demanda.
} 
la existencia y la ausencia de un análisis formal de la estabilidad del equilibrio general, el numerario puede imponerse desde un principio, ${ }^{4}$ eliminando así el arbitraje. Por esta razón no puede hablarse de una ley del numerario, es más bien un postulado del numerario. ¿Cuál es entonces el objetivo de la propuesta de este ensayo? Ésta está encaminada a reflexionar respecto de la ausencia de unicidad de precios para cada mercancía como una característica fundamental del desequilibrio económico y a exponer, al mismo tiempo, la ley del numerario con todas las limitaciones que analizaremos.

\section{La teoría del valor de Walras: el rareté como causa del valor de cambio}

Al igual que otros fundadores del pensamiento neoclásico (S. Jevons, C. Menger y Edgeworth), para Walras (1874, lección 10, p. 246) la utilidad marginal (rareté) es la causa del valor en el intercambio. Este autor arguye que el análisis de las curvas de utilidad y de los recursos (cantidades de bienes) poseídos por los agentes constituyen los datos necesarios y suficientes para establecer los precios de equilibrio. Así, a partir de esta información, este autor deriva las ofertas y demandas individuales y agregadas, de modo tal que cada parte busca, a partir del intercambio, la máxima satisfacción posible de sus deseos. Luego, a partir de las ofertas y demandas agregadas, Walras deriva los precios de equilibrio que vacían los mercados.

Si algún reproche pudiera hacerse a Walras en la formulación de su teoría de los precios, es que plantea condiciones subjetivas, por la vía del rareté, para la determinación del equilibrio, condiciones que maximizan la satisfacción de los agentes económicos en forma de ecuaciones que establecen, para cada individuo, la igualdad entre la razón de las utilidades marginales de dos bienes (tasa marginal de sustitución) y el respectivo precio relativo de éstos (costo de oportunidad). ${ }^{5}$ De aquí se desprende el hecho de que la teoría neoclásica plantee

\footnotetext{
${ }^{4}$ Es esto lo que precisamente hacen Arrow y Debreu (1954) al suponer homogeneidad de grado cero de las funciones de exceso de demanda y construir un simplex que acota el sistema de precios. También, el propio Walras lo hace cuando construye posteriormente su modelo del tanteo (véase Peláez, 2004, p. 67).

${ }^{5}$ En realidad, a partir de un principio único, la maximización de la utilidad, Walras abordó su teoría del equilibrio general competitivo mediante aproximaciones sucesivas de cuatro modelos, a saber: $i$ ) de intercambio (de dos y luego de más de dos mercancías); ii) de producción; iii) de la formación de capital o crecimiento económico, y $i v$ ) de dinero y circulación. De esta forma, él obtuvo las funciones de demanda de mercancías, de ahorro agregado y del balance de liquidez deseada en función de los precios y la tasa de interés.
} 
la regla de maximización en los términos siguientes: para maximizar la satisfacción del consumidor, el ingreso monetario de éste debe ser asignado de manera tal que el último peso gastado en cada producto comprado, produzca respecto a cada mercancía, la misma cantidad extra (marginal) de utilidad.

Sin embargo, Walras también establece condiciones objetivas de equilibrio, que terminan vaciando los mercados en forma de ecuaciones de oferta y demanda. Si bien es cierto que este autor no proporciona una demostración rigurosa de que su sistema de ecuaciones tiene una solución de equilibrio general, Walras (1874-1877 [1965], lección 11, pp. 153-163) demuestra que su sistema tiene tantas ecuaciones linealmente independientes como incógnitas y proporciona un análisis heurístico al respecto. M. Morishima (1977, p. 3) señaló que "es completamente equivocado confundir a Walras con los walrasianos contemporáneos, tanto como confundir a Marx con los marxistas. Walras no sólo estaba interesado en el rigor y la elegancia de la teoría. Sobre la base de su teoría del capital, Walras propuso la nacionalización de un número de propiedades privadas (tierra, monopolios naturales, ferrocarriles, etcétera), que conducen a una reforma impositiva, y en su teoría monetaria proporciona las bases para una reforma monetaria".

Aunque la propuesta de este autor impide soluciones más generales que sí se obtendrían con desigualdades, ${ }^{6}$ Walras impuso las condiciones necesarias para obtener los mismos resultados de la maximización de la utilidad sujeta a restricciones con desigualdades. Así, él señaló la restricción de que ningún acervo de bienes podría ser negativo después del intercambio. Para una economía con dos bienes, en el modelo de Walras (1874-1877 [1965], lección 12, p. 164) cada agente intenta la máxima satisfacción de su utilidad, cuando la razón de las utilidades marginales (tasa marginal de sustitución: rareté) iguala al precio relativo (costo de oportunidad):

$$
\frac{\frac{\partial U_{i}}{\partial x_{i}}}{\frac{\partial U_{j}}{\partial x_{j}}}=\frac{P_{j}}{P_{i}} .
$$

Sin embargo, Walras (1874-1877 [1965], lección 12, p. 294) advierte que la posibilidad de que un individuo encuentre ventajoso ofrecer toda la cantidad de uno de los bienes que posee en su dotación inicial $\left(x_{i}=0 \wedge \bar{x}_{i}>0\right)$, o bien

\footnotetext{
${ }^{6}$ Recordemos que los métodos de Kuhn-Tuker proporcionan la posibilidad de soluciones óptimas de esquina.
} 
el caso extremo, que implica una demanda absolutamente nula del otro bien $\left(x_{i}=0 \wedge \bar{x}_{i}=0\right)$. En el primer caso, el poseedor de uno de los dos bienes de la economía ofrecerá toda su dotación, siempre que el precio del bien demandado en el intercambio sea menor o igual que la razón de las utilidades marginales:

$$
x_{i}=0 \wedge \bar{x}_{i}>0 \Rightarrow \frac{P_{j}}{P_{i}} \leq \frac{\frac{\partial U_{j}}{\partial x_{j}}}{\frac{\partial U_{i}}{\partial x_{i}}} .
$$

En el segundo caso, la cantidad demandada de un bien por el poseedor de otro bien es nula siempre que el precio relativo del bien demandado sea mayor que la tasa marginal de sustitución:

$$
x_{i}=0 \wedge \bar{x}_{i}=0 \Rightarrow \frac{P_{j}}{P_{i}} \geq \frac{\frac{\partial U_{j}}{\partial x_{j}}}{\frac{\partial U_{i}}{\partial x_{i}}} .
$$

\section{LA UNICIDAD DEL PRECIO}

\section{El intercambio competitivo y la ley del numerario ${ }^{7}$}

\section{a) El equilibrio imperfecto.}

Walras generaliza la solución de su "equilibrio de intercambio esencial" de dos mercancías mediante un enfoque muy peculiar, tal que para $m$ bienes se tienen $\frac{m(m-1)}{2}$ parejas de bienes a ser intercambiadas unas entre otras y, por ende, podrá obtenerse un equilibrio para cada pareja de bienes en la misma forma que en el caso del equilibrio de intercambio esencial. En efecto, esta teoría asume una economía de $m$ bienes, $i=1, \ldots, m, h$ agentes, $\alpha=1, \ldots, h$, y formula, para el análisis del equilibrio imperfecto, una primera etapa sobre la base de dos hipótesis básicas, a saber: i) cada agente posee un sólo bien; ii) los agentes poseen información parcial sobre los precios, pues únicamente conocen la información del precio relativo del bien que poseen y que desean intercambiar respecto a otros bienes en

\footnotetext{
${ }^{7}$ El análisis de este apartado está basado en Leon Walras (1874), lección 11, parte III, así como en las discusiones que sostuve con el profesor Carlo Benetti.
} 
los mercados que se establecen para tal efecto. ${ }^{8}$ Sin embargo, Walras $(1874$, lección 12, p. 164) explicita más adelante la naturaleza de esta segunda hipótesis en los términos siguientes:

Si los participantes en el intercambio son poseedores de una única mercancía, y si, para dejar que se realicen los arbitrajes, se vocean $m(m-1)$ precios de $m$ mercancías consideradas dos a dos, no sometidos a la condición de equilibrio general, la satisfacción máxima tendrá lugar, para cada individuo, cuando los cocientes entre las raretés de las mercancías demandadas y la rareté de la mercancía que posee inicialmente, sean iguales no a los precios voceados sino a los verdaderos precios obtenidos por medio del arbitraje.

Así, por ejemplo, en el caso de una economía de tres bienes $(A, B, C)$, el agente poseedor del bien $A$ conocerá los precios relativos $P_{a, b}, P_{a, c}$, pero desconocerá el precio relativo $P_{b, c}$, que se establece en un mercado donde dicho agente no participa. Se supone que para los intercambios entre bienes existe una división en tantos mercados como pares de bienes se intercambian. Es decir, que existen $\frac{m(m-1)}{2}$ mercados, ${ }^{9}$ en cada uno de los cuales se intercambian dos bienes y se establece el correspondiente precio relativo. El intercambio del bien $A$ por el bien $B$ y del bien $B$ por el bien $A$, por ejemplo, se efectúa en el mismo mercado $(A-B)$ al precio $P_{a, b}$.

Esto supone que los agentes poseedores del bien $A$, que deseen adquirir el bien $B$ y el bien $C$, por ejemplo, simplemente acudirán con el bien $A$ a los mercados $(A-B)$ y $(A-C)$ para efectuar los respectivos intercambios en una transacción bilateral directa. En esta economía con $m$ bienes, se tienen $m(m-1)$ ecuaciones de mercado (oferta o demanda) y $m(m-1)$ incógnitas (precios relativos). Sin embargo, a pesar de tener un sistema con el mismo número de ecuaciones que de incógnitas, Walras (1874, p. 157) advierte que esta primera etapa del análisis es únicamente la mitad de la solución:

\footnotetext{
${ }^{8}$ En realidad, en primera instancia, Walras sólo explicita la primera hipótesis, como se verá más adelante, la segunda es una consecuencia de la primera. Fue Carlo Benetti (1985) quien tuvo la intuición de sugerir la necesidad de explicitar esa segunda hipótesis para el examen que haremos de la primera etapa del proceso de equilibrio imperfecto. Es necesario advertir que he recibido críticas respecto de la existencia de esta segunda hipótesis impuesta en una primera etapa analítica, ya que, en efecto, la misma no es explicitada desde un inicio por Walras. Sin embargo, ésta es la única forma en que puede funcionar el proceso que conduce al equilibrio imperfecto que este autor formula, previo a la introducción del numerario, cuando efectivamente esta hipótesis es removida.

${ }^{9} \mathrm{O}$ bien combinaciones de $m$ en $2:\left(\begin{array}{c}m \\ 2\end{array}\right)=\frac{m}{2(m-2)}$, pues el orden de los bienes que constituyen el mercado no importa: el mercado $(A-B)$ es equivalente al mercado $(B-A)$.
} 
En las condiciones definidas anteriormente, existiría en el mercado un cierto equilibrio de las mercancías tomadas dos a dos; pero no sería más que un equilibrio imperfecto. El equilibrio perfecto o general del mercado sólo tendrá lugar si una de dos mercancías cualesquiera en términos de la otra es igual al cociente entre los precios de ambas en términos de una tercera mercancía cualesquiera. ${ }^{10}$

En efecto, como consecuencia de generalizar el caso del intercambio de dos mercancias, en cada mercado existe un intercambio bilateral directo, de modo que no existe un sistema de interdependencia general, debido a que los agentes sólo poseen información de los mercados en los que actúan. En el caso más sencillo de tres bienes, $A, B$ y $C$, el poseedor del bien $A$, que desea los bienes $B$ y $C$, acude a los mercados $(A-B)$ y $(A-C)$, mientras el poseedor del bien $B$ hace lo mismo acudiendo a los mercados $(A-B)$ y $(B-C) \mathrm{y}$, por último, el poseedor del bien $C$ hace lo propio en los mercados $(A-C)$ y $(B-C)$. En estas circunstancias, la solución que ofrecen los mercados aislados no puede ser la de un sistema de precios competitivos de interdependencia general, pues cada mercado actúa de modo independiente, y donde los agentes son tomadores de un precio, pues implícitamente existe un subastador que ajusta los precios relativos en cada uno de estos mercados aislados. Por ello, aunque en cada uno de los mercados podría haber un equilibrio, Walras argumenta que éste muy probablemente sería "imperfecto".

Examinemos con más profundidad lo que nuestro autor propone cuando habla de la posibilidad de un equilibrio imperfecto. Walras señala que va a generalizar los resultados obtenidos para el precio de equilibrio en el caso de una economía de dos bienes, con el fin de aplicarlo al caso general del intercambio de varios bienes.

El equilibrio de un sistema así muy probablemente será imperfecto porque el precio relativo de una mercancía en relación con otra se establece pensando en las condiciones que son necesarias y suficientes para el equilibrio de un sistema de dos mercancías, que ya hemos señalado en la sección anterior:

$$
\frac{\frac{\partial U_{i}}{\partial x_{i}}}{\frac{\partial U_{j}}{\partial x_{i}}}=\frac{P_{j}}{P_{i}} .
$$

${ }^{10}$ Cursivas nuestras. 
En consecuencia: el intercambio de dos mercancías en un mercado regido por la libre competencia es una operación por medio de la cual todos los poseedores, tanto de una como de las dos mercancías, pueden lograr la mayor satisfacción posible de sus necesidades, con la condición de entregar la mercancía que venden y recibir la mercancía que compran en una proporción común e idéntica [...]. Los precios corrientes o de equilibrio son iguales al cociente de las raretés. ${ }^{11}$

En otras palabras: los valores de cambio son proporcionales a las raretés. (Walras, 1874, pp. 143-145.) Pero Walras advierte que ese análisis es incorrecto, en el marco de un equilibrio de interdependencia general de un sistema de intercambio de múltiples mercancías, pues esta generalización requiere que se amplíen las premisas que fueron construidas para el estudio del equilibrio de un sistema de intercambio de dos bienes. En efecto, ahora las funciones de ofertas y demandas individuales y agregadas, en cada uno de los mercados, ya no dependerán exclusivamente del precio relativo de las mercancías que se ofrecen y demandan en esos mercados, sino también de los precios relativos de los bienes de todos los demás mercados. ${ }^{12}$ Dado que cada uno de $\operatorname{los} \frac{m(m-1)}{2}$ precios relativos de equilibrio han sido establecidos pensando en mercados aislados (sistema de intercambio de dos mercancías), lo más seguro es que este sistema de precios de equilibrio de mercados aislados no desemboque en un sistema de precios de interdependencia general. Veamos ahora cómo expone Walras este problema y la solución que ofrece para un sistema de esta naturaleza.

\section{b) Intercambio indirecto, arbitraje y equilibrio general.}

En una segunda etapa analítica, Walras muestra la interdependencia general de los mercados cuando se les proporciona a los agentes la información que les faltaba, así implícitamente elimina la segunda hipótesis, ${ }^{13}$ aunque más adelante

${ }^{11}$ La idea de raretés es equivalente a las utilidades marginales. Por consiguiente, Walras está definiendo el equilibrio en términos de la maximización de la utilidad. En efecto, hoy sabemos que el resultado de este análisis formal es la igualdad entre el cociente de las utilidades marginales (tasa marginal de sustitución) con la razón de los precios o precios relativos (costo de oportunidad). Las cursivas son nuestras para subrayar el hecho de que se está levantando la segunda hipótesis.

${ }^{12}$ El establecimiento de esta interdependencia general es uno de los mayores méritos y contribuciones teóricas de Walras.

${ }^{13}$ Ahora sí existe información completa, lo cual puede adaptarse al marco de lo que posteriormente Arrow y Debreu denominaron un sistema completo de mercados. 
Walras (1876, lección 12, p. 164) lo hace explícito al señalar lo siguiente:

[...] si los participantes en el intercambio poseen inicialmente varias mercancías y si, para evitar que tengan lugar arbitrajes, se vocean los $m-1$ precios de $m-1$ mercancías en términos de la $m$-ésima tomada como numerario, teniendo en cuenta que el precio de dos mercancías cualesquiera en términos de una de la otra será igual al cociente entre los precios de una y otra en términos del numerario, es evidente que la satisfacción máxima tendrá lugar para cada individuo cuando los cocientes entre las raretés de las mercancías que no son numerario y la rareté de la mercancía utilizada como numerario sean iguales a los precios voceados.

Esto abre la posibilidad de intercambios indirectos cuando los precios no son de equilibrio, como se analizará a continuación. Por ejemplo, supóngase la siguiente estructura de precios relativos: $P_{c, a}>P_{c, b} P_{b, a} \Rightarrow P_{a, c}<P_{a, b} P_{b, c}$, que a la vez puede formularse como $P_{b, a}<P_{b, c} P_{c, a}$ y $P_{c, b}<P_{c, a} P_{a, b}$ (pueden generarse más desigualdades, pero con éstas es suficiente para el análisis de los intercambios y sus consecuencias). En términos de la estructura de precios relativos, el desequilibrio anterior es formulado por Walras de la manera siguiente: $\frac{P_{a, b} P_{b, c} P_{c, a}}{\alpha}=1$ tal que $\alpha \neq 1$. Supóngase que $P_{a, b}=8, P_{b, c}=3, P_{a, c}=6$, con lo cual se obtiene $\alpha=4$ (mayor que 1).

Primero, puede observarse que la desigualdad $P_{a, c}<P_{a, b} P_{b, c}$ implica que el agente poseedor del bien $A$ y demandante de $C$ prefiere el intercambio indirecto al intercambio directo. Esto significa que primero ofrece $A$ y demanda $B$ (en el mercado $A-B$ ), para luego acudir al mercado $(B-C)$, a ofrecer $B$ y a demandar $C$. Por tanto, en el mercado $(A-C)$ no existe oferta de $A$ ni demanda de $C$. Efectivamente, el intercambio indirecto es más favorable para aquellos poseedores del bien $A$ que demandan el bien $C$, pues en lugar de intercambiar directamente una unidad del bien $A$ por 6 unidades del bien $C$, primero obtienen 8 unidades del bien $B$ a cambio de dicha unidad del bien $A$, en el mercado $(A-B)$, para luego adquirir 24 unidades del bien $C$ por 8 unidades del bien $B$. De modo que existe una demanda suplementaria de 18 unidades de la mercancía $C$. Por ello, el precio de intercambio de una unidad de $C$ en términos de $A$ no es $\frac{1}{6}=0.167$, precio de intercambio directo, sino $\frac{1}{24}=0.042$, pues con $\frac{1}{24}$ unidades de $A$ pueden adquirirse $\frac{1}{24} \times 8=\frac{1}{3}$ unidades de $B$, en el mercado $(A-B)$, para luego intercambiar ese $\frac{1}{3}$ de $B$ en el mercado $(B-C)$, por $\frac{1}{3} \times 3=1$ unidad de $C$. 
Segundo, la desigualdad $P_{b, a}<P_{b, c} P_{c, a}$ refleja que en el mercado $(A-B)$ no existe oferta de $B$ ni demanda de $A$, pues el agente poseedor de $B$ y demandante de $A$ prefiere recurrir al intercambio indirecto, es decir, acudir al mercado $(B-C)$ a ofrecer $B$ a cambio de $C$, para posteriormente presentarse al mercado $A-C$ a ofrecer $C$ y a demandar $A$. En efecto, puede observarse que el precio de intercambio de $A$ en términos de $B$ no es 8 (precio de intercambio directo), sino $\frac{8}{4}=2$, puesto que con 2 unidades de $B$ puede acudirse al mercado $(B-C)$ para obtener inicialmente $2 \times 3=6$ unidades del bien $C$, y posteriormente intercambiar estas 6 unidades de $C$ en el mercado $(A-C)$ por $6 \times \frac{1}{6}=1$ unidad de $A$. En otras palabras, por la vía del intercambio indirecto, con 8 unidades de $B$ se pueden adquirir en el mercado $(A-C), 8 \times 3=24$ unidades de $C$, para que posteriormente con esas 24 unidades de $C$ puedan adquirirse $24 \times \frac{1}{6}=4$ unidades de $A$ en el mercado $(A-C)$. Es decir, existe una demanda suplementaria de 3 unidades de $A$.

Tercero, la desigualdad $P_{c, b}<P_{c, a} P_{a, b}$, implica que el agente poseedor de la mercancía $C$ y demandante de la mercancía $B$ no se presenta al mercado $(B-C)$, de modo que no existe oferta de $C$ ni demanda de $B$ pues éste también prefiere el intercambio indirecto de $C$ por $A$ (en el mercado $A-C$ ) y luego el intercambio de $A$ por $B$ en el mercado $(A-B)$. El precio de intercambio de $B$ en términos de $C$ no es 3 (precio de intercambio directo), sino $\frac{3}{4}=0.75$, puesto que con 0.75 unidades de $C$ puede acudirse al mercado $(C-A)$ para obtener $\frac{3}{4} \times \frac{1}{6}=\frac{1}{8}=0.125$ unidades de A, y posteriormente intercambiar esas 0.125 unidades de $A$ en el mercado $(A-B)$ por $\frac{1}{8} \times 8=1$ unidad de $B$. Por consiguiente, también resulta más favorable el intercambio indirecto de $C$ por $B$, ya que con 3 unidades de $C$ pueden adquirirse $3 \times \frac{1}{6}=\frac{1}{2}=0.5$ unidades de $A$ en el mercado $(C-A)$, y luego, con esas 0.5 unidades de $A$ comprar $\frac{1}{2} \times 8=4$ unidades de $B$ en el mercado $(A-B)$. Entonces, también existe una demanda suplementaria de 3 unidades de $B$.

Walras asume que este desequilibrio, originado por la diferencia entre precio relativo directo e indirecto, ${ }^{14}$ genera un proceso estable de variación de los precios relativos, tal que una demanda excedente de una mercancía en términos de otra conlleva un incremento del precio relativo y viceversa. A este desequilibrio Walras lo denomina "arbitraje": preferencia de los intercambios indirectos sobre los intercambios directos, y cuyo resultado es un bloqueo de los intercambios, pues en los mercados $(A C),(A B) y(B C)$ no hay intercambios. Sabemos que el trueque bloquea los intercambios, ${ }^{15}$ lo interesante de este análisis es que Wal-

\footnotetext{
${ }^{14}$ Ausencia de unicidad de precios.

${ }^{15}$ Para un excelente análisis al respecto véase C. Benetti (1990, pp. 23-38).
} 
ras está mostrando que el desequilibrio generado por la ausencia de unicidad de precio para cada mercancía también bloquea los intercambios, lo cual constituye la premisa de la proposición de este autor, de acuerdo con la cual fuera del equilibrio no es posible el intercambio.

En virtud de que $P_{a, b} P_{b, c} P_{c, a}>1$, i.e., $\frac{P_{a, b} P_{b, c} P_{c, a}}{\alpha}=1$ tal que $\alpha>1$, resulta evidente que las operaciones de arbitraje conducirán a que $P_{b, a}$ tienda a subir por exceso de demanda de la mercancía $A$ en el mercado $(A-B)\left(P_{a, b}\right.$ disminuye). Por las mismas razones $P_{c, b}$ tenderá a incrementarse por la demanda suplementaria de $B$ en términos de $C$ ( $P_{b, c}$ disminuye). Por las mismas circunstancias, en el mercado $(A-C) P_{a, c}$ tenderá a la alza $\left(P_{c, a}\right.$ bajará). Las ganancias que obtienen los agentes con estas operaciones de arbitraje las distribuyen en función de sus preferencias, adquiriendo un poco más de uno u otro bien con el fin de obtener la máxima satisfacción (maximizar su utilidad), es decir, que la utilidad marginal llega a igualar a los precios relativos de las operaciones de arbitraje (precios relativos de intercambio indirecto) que desembocan en las demandas suplementarias que hemos analizado. Aunque no se ha examinado el caso en que $\alpha<1$, o sea, $P_{b c}<\frac{P_{b a}}{P_{c a}}$, los resultados serían a la inversa de lo que hemos obtenido y las operaciones de arbitraje conducirían a una alza de $P_{a, b}$, una caída de $P_{a, c}$ y una alza en $P_{b, c}$. En una u otra dirección $(\alpha>1$ o $\alpha<1)$, se tendrá una situación de desequilibrio ${ }^{16}$ caracterizada por una variación de los precios relativos.

Al mismo tiempo que este autor asume un proceso estable de variación de los precios, formula el siguiente sistema de ecuaciones que verifica el equilibrio general (perfecto), tal que se puede examinar directamente una economía, donde los precios están expresados en un numerario común, la mercancía $A$ en este caso:

$$
\begin{gathered}
P_{a, b}=\frac{1}{P_{b, a}}, P_{c, b}=\frac{P_{c, a}}{P_{b, a}}, P_{d, b}=\frac{P_{d, a}}{P_{b, a}}, \ldots \\
P_{a, c}=\frac{1}{P_{c, a}}, P_{b, c}=\frac{P_{b, a}}{P_{c, a}}, P_{d, c}=\frac{P_{d, a}}{P_{c, a}}, \ldots \\
P_{a, d}=\frac{1}{P_{d, a}}, P_{b, d}=\frac{P_{b, a}}{P_{d, a}}, P_{c, d}=\frac{P_{c, a}}{P_{d, a}}, \ldots
\end{gathered}
$$

\footnotetext{
${ }^{16}$ Desequilibrio que consiste en la existencia de dos precios relativos para cada mercancía, un precio relativo de intercambio directo y un precio relativo que aprovecha las oportunidades de arbitraje del intercambio indirecto.
} 
Se trata de un sistema de $(m-1)$ ecuaciones de equilibrio general, con $\alpha=1$, lo cual implica que se obtienen las mismas cantidades de bienes por medio del intercambio directo o por intercambio indirecto: unicidad del precio de intercambio. El bien en términos del cual los precios de todos los otros bienes son expresados ( $A$ en este caso), es denominado por Walras como numerario. ${ }^{17} \mathrm{En}$ este caso, el precio relativo de un bien es único (lo cual implica ausencia de arbitraje), pues el precio de $C$ en términos de $A$, por ejemplo, es igual en el intercambio directo que en el intercambio indirecto. En el caso de intercambio indirecto, dado que el precio de $C$ en términos de $B$ es $P_{c, b}$, y dado que con $P_{c, b}$ unidades de $B$ es posible en primera instancia la obtención de $P_{c, b} P_{b, a}$ unidades del bien $A$ en el mercado $(A-B)$, al precio de $A$ en términos de $B, P_{a, b}=\frac{1}{P_{b, a}}$, para luego intercambiar estas $P_{c, b} P_{b, a}$ unidades de $A$ en el mercado $(A-C)$, por $P_{c, b} P_{b, a} P_{a, c}=1 \mathrm{de}$ $C$ al precio de $C$ en términos de $A P_{c, a}=\frac{1}{P_{a, c}}$.

\section{c) Los bienes gratuitos}

En el marco del análisis del intercambio que hace Walras, aparentemente los bienes gratuitos no tienen ningún lugar, sin embargo, esto no es del todo cierto y por esa razón introducimos este apartado. En efecto, se afirma que el análisis riguroso de los bienes gratuitos se inicia con los trabajos simultáneos de tres economis$\operatorname{tas}^{18}$ alemanes, a saber: Zeuthen (1932), Neisser (1932) y Von Stackelberg (1933). Pero hay que recordar que la idea principal de la teoría del intercambio de Walras se fundamenta en que los bienes tienen valor (1874, pp. 115 y 145), y se intercambian por ser útiles y viceversa, y analiza entonces la demanda individual de un bien gratuito:

...la cantidad efectivamente demandada de $(A)$ por este individuo a un precio nulo, es decir la cantidad que consumiría si la mercancía $(A)$ fuese gratuita $[\ldots]$ No podría existir última necesidad satisfecha si no hubiese necesidad, si la mer-

${ }^{17}$ W. Jaffê, traductor de la obra de Walras (Elements of Pure Economics, 1954, p. 515) señala que el término numerario no se puede traducir para ser asociado con el uso especial que Walras le da. Jaffê arguye que probablemente Walras fue el primer autor en emplear esta expresión para designar una mercancía que en todos los otros aspectos es igual a todas las demás, excepto que también sirve como un bien estándar en términos del cual todos los demás bienes son expresados. John Hicks (1946, p. 58), inspirado en Walras le denominó mercancía estándar, pero la bibliografía contemporánea le llama numerario en el mismo sentido.

${ }^{18}$ Véase Morishima (1977, p. 14); Arrow y Hahn (1971, p. 9) hacen un análisis más detallado de la aportación de estos tres autores. 
cancía no tuviese utilidad intensiva ni extensiva, si fuese inútil. Y la intensidad de la última necesidad satisfecha sería nula si la mercancía, teniendo curva de utilidad, existiera en cantidad superior a su utilidad extensiva, si fuese ilimitada en cantidad.

Con este propósito, Walras hace hincapié particular en la cantidad demandada cuando el precio del bien es nulo. Dicha cantidad, normalmente finita, es denominada utilidad extensiva de dicho bien. La utilidad extensiva agregada de un bien $i$, sumada sobre todos los individuos, viene a ser la cantidad de $i$ que los agentes desean retener cuando su precio es cero. Puesto que cada demanda individual de $x_{i}$ depende no sólo de $P_{i}$, sino también de todos los otros precios, entonces, el agregado $X_{i}$ es una función de todos los precios, de modo que la utilidad extensiva total, cuando $P_{i}=0$, fluctúa si los precios de los otros bienes cambian.

Las demostraciones contemporáneas de existencia del equilibrio se definen en términos de la posibilidad de que existan bienes gratuitos. Walras, por su parte, examina la existencia del equilibrio de intercambio en términos de que la demanda de cada bien sea menor o igual a la oferta. Por ello, nos parece pertinente el argumento de Morishima (1977, p. 17):

Un equilibrio de intercambio es un equilibrio Arrow-Debreu, pero lo contrario no. Si llamamos al primero un equilibrio de intercambio esencial, podemos decir que lo que Walras quiso descubrir es si el equilibrio Arrow-Debreu es esencial o no; su respuesta a esta pregunta fue negativa, a menos que fuesen impuestas condiciones adicionales. ${ }^{19}$

\section{CONCLUSIONES}

Esta teoría del desequilibrio es única en su género respecto de la ausencia de un único precio relativo para cada mercancía; nadie después de Walras se ocupó del

\footnotetext{
${ }^{19}$ Este problema de la esencialidad no es patrimonio de una economía de intercambio. Si en una economía con producción no se produce ningún bien, el equilibrio se reduce a un equilibrio de intercambio: equilibrio de producción sin producción. De la misma manera, se puede estar frente a un equilibrio de acumulación sin acumulación, o un equilibrio monetario de una economía no monetaria. De estos ejemplos, únicamente el último es famoso: el problema Hahn. Sin embargo, el problema va más allá de la esencialidad; se trata también de un problema de existencia. Una excelente discusión del problema Hahn la encontramos en Benetti (1989, pp. 100-104).
} 
estudio del numerario. En efecto, el arbitraje está constituido por la ausencia de unicidad de precios relativos de las mercancías, tal que resulta más favorable el intercambio indirecto, es decir, el intercambio en desequilibrio, aunque hay que advertir que este último resulta teóricamente virtual puesto que fuera del equilibrio las transacciones se bloquean. Precisamente la existencia de demandas suplementarias originadas por el arbitraje constituye la expresión y el resultado del desequilibrio. Para resolver este desequilibrio, Walras intuye un proceso cualitativo de ajuste de precios: suben los precios relativos de las mercancías con demandas suplementarias y bajan los precios relativos inversos respectivos.

Resulta evidente que, para poder entender el equilibrio imperfecto de mercados aislados, se requiere analizar el equilibrio que Walras examina y propone para el caso de dos mercancías que se intercambian una por otra. Walras plantea dos etapas analíticas: primero el equilibrio imperfecto de mercados aislados, luego el arbitraje que desemboca simultáneamente en el numerario y el equilibrio general. Los límites de la primera etapa están constituidos por las dos hipótesis ad hoc que hemos analizado.

Una prueba sobre la necesidad de estas hipótesis, en la primera etapa, es muy sencilla y alcanza, sin pérdida de generalidad, con seguir el ejemplo de una economía de tres mercancías: $A, B$ y $C$. ¿Qué ocurre si un agente posee dos bienes?, $A$ y $B$, por ejemplo. Es evidente que ahora este agente conoce toda la estructura de precios relativos: $P_{a, b}, P_{a, c} y P_{b, c}$. En consecuencia, las dos hipótesis iniciales no se verifican.

En estas nuevas condiciones, el equilibrio imperfecto no existe debido a que los agentes plenamente informados se percatan de la existencia o no de oportunidades de arbitraje desde un principio. Si estas oportunidades existen se presentarán en algunos mercados y en otros no, para aprovechar las ventajas del intercambio indirecto. Como ya lo hemos mostrado, esto bloquea las transacciones, a la vez que la desigualdad entre oferta y demanda produce una variación en los precios. Por consiguiente, el análisis de Walras carece de generalidad, pues el equilibrio imperfecto es consecuencia de esas dos hipótesis ad hoc. Si éstas se eliminan, entonces se impone el arbitraje desde un principio. Un problema adicional se plantea respecto de la hipótesis de la dotación inicial de un solo bien. En efecto, ésta tendría que ser tal que pudiese alcanzar para el financiamiento de los intercambios de todos los otros bienes deseados. Tampoco se especifica si cada tipo de agente posee la misma dotación. Esto podría generar problemas con el ajuste de las cantidades. 
En la segunda etapa, el arbitraje trae como consecuencia la variación de los precios. Al respecto, Walras se limita a indicaciones cualitativas acerca del sentido de dicha variación, pero no proporciona ningún análisis dinámico de la estabilidad del equilibrio general de los mercados. Este equilibrio se establece simultáneamente con un sistema de precios en términos de numerario, en el cual queda eliminado el problema del arbitraje. En ese sentido podría hablarse de una ley económica: ${ }^{20}$ el numerario como resultado de un proceso económico, pero sin una demostración de estabilidad. Por consiguiente, Walras tiene el gran mérito de haber planteado el problema de la unicidad del precio pero no lo resolvió. Sin embargo, John Hicks (1946, Cap. V, pp. 62-77), mediante un supuesto de regularidad analizó las condiciones de estabilidad para la economía de dos bienes propuesta por Walras y generalizó, inspirado en la ley de la oferta y la demanda de éste, dichas condiciones para el caso de una economía con múltiples bienes. ${ }^{21}$

La ley del numerario se justifica sólo si el proceso de arbitraje es estable, pero Walras no proporciona ninguna solución al respecto, ya que el numerario se impone arbitrariamente en el análisis del desequilibrio. Este problema no ha sido estudiado después de Walras, al extremo que hoy en día la teoría del equilibrio general evade este problema, imponiendo arbitrariamente un numerario en el estudio del equilibrio, pero, lo que es aún más grave, también lo hace en el estudio del desequilibrio. Al respecto, es digno de señalar lo que plantean K. Arrow y F. Hahn (1971, p. 322): “...los procesos investigados... suponen que, a pesar del desequilibrio, hay sólo un precio para cada bien en todo momento... Sabemos que es difícil el análisis del comportamiento fuera de equilibrio de una economía donde ocurren transacciones a todos los precios". ${ }^{22}$

Una objeción a lo que hemos expuesto podría estar fundada en el hecho de que estamos presentando analíticamente la presencia del numerario en el marco de un problema de información. ${ }^{23}$ Así, podría argumentarse que en realidad el numerario sencillamente es consecuencia de la homogeneidad de grado cero en

\footnotetext{
${ }^{20}$ Véase Benetti (1985).

${ }^{21}$ En términos técnicos, esta condición del equilibrio perfectamente estable de Hicks (1946, anexo matemático, pp. 315-319) dice que el signo de los menores principales del jacobiano de derivadas parciales de las funciones de exceso de demanda se alterna (en el caso de una economía de dos bienes esta condición hicksiana coincide con la de Walras). Puesto que esta condición de estabilidad se formuló sin examinar plenamente los procesos dinámicos de ajuste de los mercados, encontró su mayor utilidad en la estática comparativa (véase Peláez, 2008).

${ }^{22}$ Cursivas nuestras.

${ }^{23}$ En virtud de la segunda hipótesis dentro de la primera etapa que examina el equilibrio imperfecto.
} 
precios y renta de las funciones de exceso de demanda, tal que el valor de la renta de la dotación inicial de cada agente depende del sistema de precios vigente. El argumento que está detrás de esta objeción es precisamente la ley de Walras: la suma del valor de la demanda es financiada con la suma del valor de la oferta, pues estamos en una economía donde no existe el robo ni el regalo.

Efectivamente, es importante advertir que un supuesto matemático clave para la demostración de existencia del equilibrio general que ofrecen Arrow y Debreu, es que las funciones de demanda y de excesos de demanda, individuales y agregadas, son homogéneas de grado cero en precios y renta: $x_{i}(\lambda P, \lambda W)=x_{i}(P$, $W) \forall i \& Z_{i}(\lambda P, \lambda W)=Z_{i}(P, W) \forall i$. Una de las implicaciones de la Ley de Walras, formulada por Oscar Lange, es la existencia de $m-1$ ecuaciones linealmente independientes en un modelo de equilibrio general con $m$ mercancías, es decir, si m-1 mercados están en equilibrio, el m-ésimo mercado también estará en equilibrio. Entonces ¿cómo resolver un sistema de $m$ precios con sólo $m-1$ ecuaciones? La respuesta está en que existen únicamente $m-1$ precios linealmente independientes.

Por tanto, si existe un vector de precios de equilibrio $P^{*}=\left(P_{1}^{*}, P_{2}^{*}, \ldots, P_{i}^{*}, \ldots, P_{m}^{*}\right)$, tal que $\lambda=\frac{1}{P^{*}}$, evidentemente estamos eligiendo uno de los precios, $\left(P_{i}^{*}\right)$, como numerario, es decir: $\lambda P_{i}^{*}=1$, tal que los $m-1$ precios restantes se interpretan como precios relativos en términos de éste, y es por eso que dicho precio se interpreta como numerario. ${ }^{24}$ Lo que hizo Arrow y Debreu es, entonces, formalizar la teoría del numerario de Walras; evidentemente, Walras construye este concepto teórico sobre la base de sugerir hipótesis económicas, como debe ser. Lo que hemos observado en los pasados sesenta años ha sido al revés, pues el método axiomático nunca se ha visto obligado a conectar los postulados con los conceptos y razonamientos de la teoría económica. Walras primero construyó el postulado del numerario y luego obtuvo las consecuencias teóricas, los primeros en reconocer la paternidad de este autor en la formulación del numerario son Hicks, Samuelson, Hahn, Arrow y Debreu, etcétera. Koopmans (1957, pp. 187-197) señala que:

\footnotetext{
${ }^{24}$ Arrow y Debreu normalizan los precios para expresarlos en términos de precios relativos, tal que ${ }^{\lambda=\frac{1}{m}} \sum_{i=1}^{m} P_{i}$. Cada precio se reemplaza por su precio normalizado $P_{i}^{P_{i}}=\frac{P_{i}}{\sum_{i=1}^{m} P_{i}}$. La consecuencia es que la suma de todos los precios relativos así normalizados es igual a uno, $\sum_{i=1}^{m} P_{i}^{\prime}=1$. Por consiguiente, se puede restringir la atención al vector de precios relativos que pertenece al simplex $m-1$ dimensional: $S^{m-1}=\left\{P \in \mathbf{R}^{m}: \sum_{i=1}^{m} P_{i}^{\prime}=1\right\}$ que por ser un conjunto no vacío, compacto y convexo, al asumir un mapeo funcional continuo $f: S^{m-1} \rightarrow S^{m-1}$ puede aplicarse el teorema del punto fijo para demostrar la existencia del equilibrio.
} 
Se ha apelado a la equivalencia lógica esencial entre las matemáticas y el lenguaje (Samuelson), junto a la mayor eficacia y concisión del razonamiento matemático en numerosos problemas importantes (Samuelson, Stigler y otros) En lo que respecta a la relación entre los supuestos y el razonamiento, se ha reconocido generalmente que la aplicación correcta del método matemático fuerza al investigador a ofrecer una presentación completa de hipótesis garantizadamente no contradictorias (Marshak, Samuelson) [...] al no tener símbolos los matemáticos ningún significado natural más que el que les atribuyen los postulados o definiciones, las connotaciones de las palabras en el lenguaje común no pueden interferir con el proceso de razonamiento. Sin embargo [...] no hay nada en el método matemático que obligue a los conceptos postulados con entidades observables. [...] La dificultad en economía dinámica ha consistido en que los instrumentos han sugerido qué hipótesis hacer, y no al contrario. ${ }^{25}$

En estas circunstancias, Walras considera haber obtenido el numerario como resultado de un proceso económico (ley del numerario) y pasa a un segundo modelo de mercados interdependientes con numerario desde un principio, al suprimir las hipótesis ad hoc que utilizó en la teoría del equilibrio imperfecto, con lo cual se elimina el problema del arbitraje. Se trata de su célebre teoría del tâtonnement que nos introduce en el problema de la estabilidad. Este modelo lo hemos examinado en otro ensayo (Peláez, 2004, pp. 61-80).

La salida propuesta por Walras al problema del equilibrio imperfecto, su teoría del arbitraje y la ley del numerario, conduce a un dilema para la teoría económica, el cual es abordado por M. Morishima (1977). En efecto, por una parte, Walras propone el modelo de arbitraje, mediante trueque directo de dos agentes donde emergen precios relativos que constituyen una tasa de intercambio ex post entre parejas de bienes, sin una teoría de formación de precios, modelo que hemos estudiado en este ensayo. ${ }^{26}$ Pero, al mismo tiempo, Walras propone un modelo de intercambio individual con agentes tomadores de precios, en términos de un numerario, que son propuestos y ajustados por un subastador. Se trata, entonces, de dos modelos basados en dos conceptos distintos de precios. Morishima (1977, pp. 20-26) arguye que ni es evidente ni claro que ambos modelos generen el mismo equilibrio general, al tiempo que abre tres interrogantes, a saber:

\footnotetext{
${ }^{25}$ Cursivas nuestras.

${ }^{26}$ Podría asumirse la propuesta de formación de precios de Menger (1871, Cap. V, pp. 170198), adecuada al rareté de Walras.
} 
i) ¿Existe un equilibrio para el modelo con subastador? y/o

ii) ¿Existe un equilibrio para el modelo con arbitraje? Si la respuesta es afirmativa en uno y otro caso:

iii) ¿Son ambos equilibrios generales equivalentes? Si la tercera interrogante se resuelve es evidente que no se requiere una demostración de existencia para ambos casos. ${ }^{27}$

Finalmente, Arrow y Hahn (1971, p. 5) apuntan que la meta de Walras era aún más ambiciosa en términos de su análisis del equilibrio general, ya que se dirigía a la variación de los precios cuando se modifican los fundamentales de la economía: "[...] Walras buscaba un objetivo todavía más elevado con su análisis del equilibrio general: quería estudiar lo que ahora se llama estática comparativa; en otras palabras, las leyes de variación de los precios y cantidades de equilibrio al variar los datos básicos (recursos, condiciones de la producción o funciones de utilidad)".

Este desafío tuvo que esperar más de medio siglo; fueron las aportaciones de Hicks (1946) y Samuelson (1937) las que permitieron decir algo al respecto. Gracias a los trabajos de Sonnenschein (1972, 1973a, 1973b), Mantel (1974) y Debreu, (1974) que se resumen en lo que se denomina Teorema Sonnenschein-Mantel-Debreu (Peláez, 2008 y 2009), ahora sabemos que esta tarea se imposibilita, en virtud de la ausencia de unicidad del equilibrio general competitivo inspirado en Walras.

\footnotetext{
${ }^{27}$ Morishima (1977, p. 21) hace una demostración y concluye que no se requiere una demostración de existencia para el equilibrio que surge de la ley del numerario. Sin embargo, él se aparta de la hipótesis de Walras, según la cual cada agente posee una dotación inicial de un solo bien, pues asume una dotación inicial donde es posible que tengan cabida todos los bienes. Ahora sabemos que es imposible resolver con precisión este dilema, pues en virtud del Teorema Sonnenschain-MantelDebreu (véase Peláez, 2008), el modelo del tanteo propuesto por Walras es inestable y genera múltiples equilibrios.
} 


\section{REFERENCIAS BIBLIOGRÁFICAS}

Arrow, Kenneth (1974), “General Economic Equilibrium: Purpose, Analytic Techniques, Collective Choice", The American Economic Review, Vol. 64, Núm. 3.

— , y L. Hurwicz (1958), "On the Stability of the Competitive Equilibrium, I", Econometrica, 26, pp. 522-552.

, H. D. Block y Leonid Hurwicz (1959), "On the Stability of the Competitive Equilibrium, II”, Econometrica, 27, pp. 82-109.

_ , y H. Hahn Frank (1971), General Competitive Equilibrium, Advanced Textbooks in Economics, Vol. 12, Editors C. J. Bliss y M. D. Intriligator, North Holland.

— , y Gerard Debreu (1954), "Existence of Equilibrium for a Competitive Economy", Econometrica, Vol. 22, Núm. 3, pp. 265-290.

Benetti, Carlo (1985), "Economie monétaire et économie de troc: la question de l'unité de compte commune", Économie Appliquée (1).

— (1990), Moneda y teoría del valor, Fondo de Cultura Económica, México.

Debreu, Gerard (1974), "Excess Demand Functions", Journal of Mathematical Economics, Vol. I, pp. 15-21.

Hahn, Frank (1982), "Stability”, en Arrow, K. y M. Intrilligator (ed), Handbook of Mathematical Economics, Vol. II, Chapter 16, pp. 745-793.

Hicks, John. R. (1946), Value and Capital (2a. ed.), Oxford University Press.

Jevons, W. Stanley (1888), The Theory of Political Economy, con una introducción de R.

D. Collison Black, Penguin Books Inc., 1970.

Koopmans, T. C. (1957), Tres ensayos sobre el estado de la ciencia económica, Antoni Bosch, editor, 1980.

Mantel, R. (1974), "On the Characterization of Aggregate Excess Demand", Journal of Economic Theory 7, pp. 348-353.

- (1976), "Homothetic Preferences and Community Excess Demand Functions", Jounal of Econoic Theory, Vol. 12, pp. 197-201.

Menger, Carl (1871), Principios de economía política, Unión Editorial, S. A., Biblioteca de Economía, 1996.

Morishima, Michio (1977), Walras Economics, A pure theory of capital and money, Cambridge University Press.

Negishi, Takashi (1962), "The Stability of a Competitive Economy: a Survey Article", Econometrica, Vol. 30, pp. 635-669. 
Peláez, José Guillermo (2004), "El tanteo de Leon Walras y la hipótesis diagonal dominante", Economía, Teoría y Práctica, Nueva Época, Núm. 21, Universidad Autónoma Metropolitana, México, pp. 61-80.

- (2006), Walras's Tantonement and the Diagonal Dominant Hypothesis, $33^{\text {rd }}$ Annual Meeting of the History of Economics Society, Grinnell College, Grinnell, Iowa, EU.

(2008), Sonnenschain-Mantel-Debreu Theorem (S-M-D-T) and its Implications into Walras's Tanonnenment, $35^{\text {th }}$ Annual Meeting of the History of Economics Society.

- (2008), Teorema Sonnenschain-Mantel-Debreu. Temas selectos de investigación económica latinoamericana, Facultad de Ciencias Económicas, Universidad de Guayaquil, Ecuador.

Samuelson, P. A. (1947), Foundations of Econmic Analysis, Cambridge University Press.

Sonnenschein, H. (1972), “Market Excess Demand Functions”, Econometrica, Vol. 40, pp. 549-563.

_ (1973a), "Do Walras' Identity and Continuity Characterize the Class of Community Excess Demand Functions?", Journal of Economic Theory, Núm. 6, pp. 345-354.

_ (1973b), "The Utility Hypothesis and Market Demand Theory", Western Economic Journal, pp. 404-410.

Walras, Leon (1874-1877), Elements of Pure Economics or the Theory of Social Wealth, traducido por William Jaffé (1954), Richard D. Irwin, Inc, segunda impresión, 1965. 
